\title{
Cervical screening in young women aged 20-24 years
}

\section{Alison N Fiander}

\section{Background}

Although the overall incidence of cervical cancer has fallen dramatically since the introduction of the systematic call-recall screening programme in 1988, averting an epidemic of cervical cancer, ${ }^{1}$ the incidence appears to be increasing in young women aged 15-44 years from 10.7/ 100000 in 1979 to $14.7 / 100000$ in 1990. Two peaks are seen in cervical cancer incidence: one for women in their 80s and the other for women in their 30s. These cohorts are at high risk and represent young women becoming sexually active following World Wars 1 and 2 and in the 1960s with the advent of oral contraception, the sexual revolution and consequent changes in sexual behaviour. Whereas the former cohort will disappear, there is no indication that the second peak will see a reduction in exposure to the central causative agent of anogenital carcinogenesis, high-risk human papillomavirus (HPV) infection.

The incidence of cervical intraepithelial neoplasia grade 3 (CIN3) has been increasing since the late 1980s in young women aged less than 35 years, whilst the same increase has not been observed for women over 35 years. ${ }^{2}$ If we assume a conservative progression rate for CIN 3 to invasion of $1 \%$ per year - and there is some suggestion that progression may be higher in younger women - significant numbers of young women are at risk of developing invasive cervical cancer, threatening future fertility if not young lives. Although a recent systematic review and metaanalysis has shown an increased risk of preterm labour following treatment for CIN, this did not significantly affect neonatal outcome. ${ }^{3}$

\section{Existing cervical screening programmes}

In Wales, cervical screening commences at age 20 years. Since the introduction of the organised screening programme in 1988, the incidence of cervical cancer in younger women has halved. ${ }^{4}$ Given that we see between 300 and 350 cases of CIN3 per year in women aged 20-24 years we might expect to see up to 15-17 cases of invasive disease in this age group if screening commenced at 25 years of age.

At the same time the English cervical screening programme has raised the age at which it commences screening, whilst attendance for screening is falling. Overall the number of eligible women screened in England within the last 5 years has fallen from $82 \%$ in 1997 to $79 \%$ in 2006/2007. 5 The levels of attendance have been declining even faster among younger women and fell below $70 \%$ in the 25-29-year age group in 2005/2006. There may be several reasons for the decline in screening coverage in young women including: embarrassment and fear over what is perceived as an invasive screening test; lack of time in busy lives while cervical cancer is relatively rare (a victim of the cervical screening programme's own success?); poor knowledge of cervical cancer and cervical screening; lack of habit and early reminders of the need for cervical screening; and a general disengagement from preventative health measures. This, combined with peak prevalence for

\section{J Fam Plann Reprod Health Care 2008; 34(1): 19}

\section{Department of Obstetrics and Gynaecology, Wales College of Medicine, Cardiff University, Cardiff, UK}

Alison N Fiander, DM, FRCOG, Professor

Correspondence to: Professor Alison Fiander, Department of Obstetrics and Gynaecology, Wales College of Medicine, Cardiff University, Heath Park, Cardiff CF14 4XN, UK.

E-mail: fianderan@cf.ac.uk cervical cancer for women in their 30s, risks leaving screening too late to pick up precursor disease, women presenting instead with symptoms of invasive disease.

The paper by Sasieni and colleagues ${ }^{6}$ demonstrated less protection for younger women from a normal cervical smear than for older women and paved the way for an increase in the age of first invitation to cervical screening in England. Sasieni's study did not include microinvasive cancers, for which fertility-sparing options for treatment may be feasible - an advantage of early screen-detected tumours. If we accept that protection from current cervical screening is poor in young women then perhaps the response to this should not have been to start screening later but to find a better method of screening young women?

\section{Future cervical screening strategies}

Screening with HPV testing alone would be problematic since up to $40 \%$ of $20-24$-year-olds might be expected to be HPV-positive with evidence of HPV infection and lowgrade cytological abnormalities on 'reflex' cervical cytology. A secondary test is therefore required for younger women to improve the specificity of HPV testing. Further work on biomarkers or prognostic indicators for underlying high-grade disease or the potential to develop high-grade disease is urgently required. Biomarker research is still in its infancy with small studies investigating the utility of markers such as p16INK4, mRNA, MCM, TOP2A, methylation, and so on. Ultimately we might expect to see automated HPV and biomarker testing, generating an individual risk for high-grade disease indicating the need for further investigation.

Screening young women also provides an opportunity for education about HPV: that it is a ubiquitous infection with up to $80 \%$ of women infected at some point in their lives. This could help dispel stigma and reduce the psychological sequelae of screening positive. Key messages about HPV infection have been defined $^{7}$ and will need further elaboration during implementation of a national HPV vaccination programme, including the need to continue with cervical screening for the foreseeable future. Just because screening is less effective in younger women are we at risk of 'throwing out the baby with the bath water'?

\section{Statements on funding and competing interests}

Funding None identified.

Competing interests None identified.

\section{References}

1 Peto J, Gilham C, Fletcher O, Matthews FE. The cervical cancer epidemic that screening has prevented in the UK. Lancet 2004; 364: 249-256.

2 Cancer Research UK. UK Cervical Cancer Incidence Statistics. 2007. http://info.cancerresearchuk.org/cancerstats/types/cervix/ incidence/ [Accessed 8 November 2007].

3 Kyrgiou M, Koliopoulos G, Martin-Hirsch P, Arbyn M, Prendiville W, Paraskevaidis E. Obstetric outcomes after conservative treatment for intraepithelial or early invasive cervical lesions: systematic review and meta-analysis. Lancet 2006; 367 : 489-498.

4 Rieck GC, Tristram A, Hauke A, Fielder H, Fiander AN. Cervical screening in 20-24-year olds. J Med Screen 2006; 13: 64-71; discussion 62-63.

5 NHS Cancer Screening Programmes. NHS Cervical Screening Programme Statistical Bulletin (England). 2007. http://www.cancerscreening.nhs.uk/cervical/statistics.html [Accessed 13 November 2007].

6 Sasieni P, Adams J, Cuzick J. Benefit of cervical screening at different ages: evidence from the UK audit of screening histories. Br J Cancer 2003; 89: 88-93.

7 Tristram A. HPV information needs. Best Pract Res Clin Obstet Gynaecol 2006; 20: 267-277. 